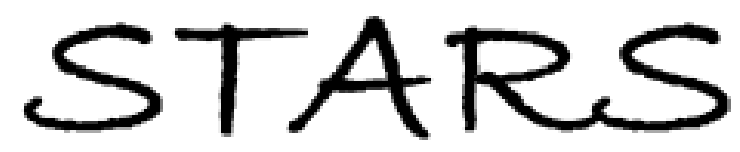

University of Central Florida

STARS

$1-1-2003$

\title{
Pore size distribution, survival probability, and relaxation time in random and ordered arrays of fibers
}

Manolis M. Tomadakis

Teri J. Robertson

University of Central Florida

Find similar works at: https://stars.library.ucf.edu/facultybib2000

University of Central Florida Libraries http://library.ucf.edu

This Article is brought to you for free and open access by the Faculty Bibliography at STARS. It has been accepted for inclusion in Faculty Bibliography 2000 s by an authorized administrator of STARS. For more information, please contact STARS@ucf.edu.

\section{Recommended Citation}

Tomadakis, Manolis M. and Robertson, Teri J., "Pore size distribution, survival probability, and relaxation time in random and ordered arrays of fibers" (2003). Faculty Bibliography 2000s. 4070.

https://stars.library.ucf.edu/facultybib2000/4070

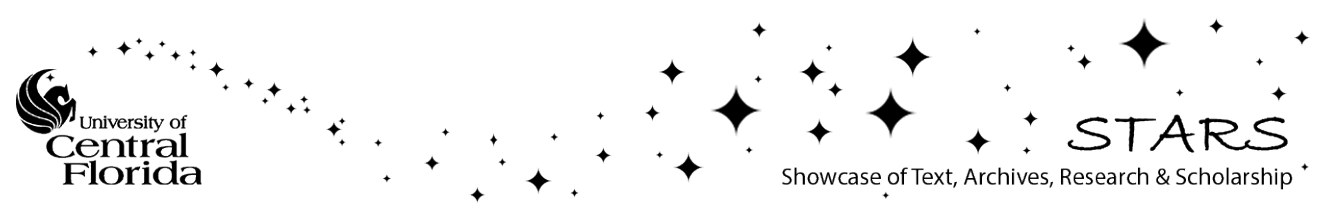




\section{Pore size distribution, survival probability, and relaxation time in random and ordered arrays of fibers}

Cite as: J. Chem. Phys. 119, 1741 (2003); https://doi.org/10.1063/1.1582431

Submitted: 11 February 2003. Accepted: 22 April 2003. Published Online: 02 July 2003

Manolis M. Tomadakis, and Teri J. Robertson

\section{ARTICLES YOU MAY BE INTERESTED IN}

Survival and relaxation time, pore size distribution moments, and viscous permeability in random unidirectional fiber structures

The Journal of Chemical Physics 122, 094711 (2005); https://doi.org/10.1063/1.1854130

Transport properties of random arrays of freely overlapping cylinders with various orientation distributions

The Journal of Chemical Physics 98, 616 (1993); https://doi.org/10.1063/1.464604

Effective diffusivities and conductivities of random dispersions of nonoverlapping and partially overlapping unidirectional fibers

The Journal of Chemical Physics 99, 9820 (1993); https://doi.org/10.1063/1.465464

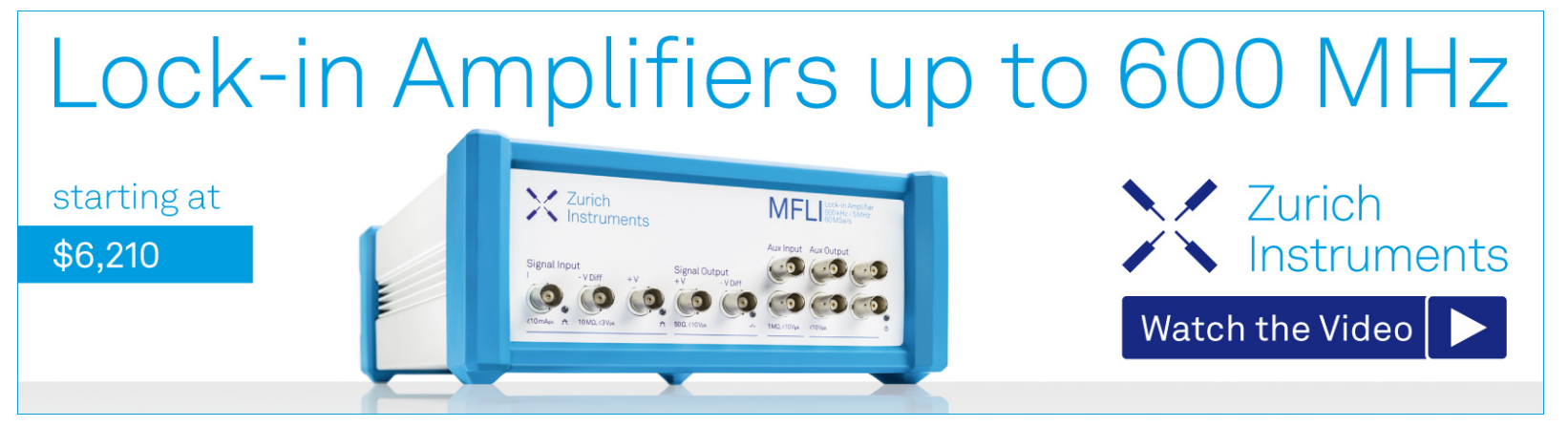

J. Chem. Phys. 119, 1741 (2003); https://doi.org/10.1063/1.1582431

119, 1741

(c) 2003 American Institute of Physics. 


\title{
Pore size distribution, survival probability, and relaxation time in random and ordered arrays of fibers
}

\author{
Manolis M. Tomadakis \\ Department of Chemical Engineering, Florida Institute of Technology, Melbourne, Florida 32901-6975 \\ Teri J. Robertson \\ Hydrogen R\&D Division, Florida Solar Energy Center, University of Central Florida, Cocoa, \\ Florida 32922
}

(Received 11 February 2003; accepted 22 April 2003)

\begin{abstract}
We present a random walk based investigation of the pore size probability distribution and its moments, the survival probability and mean survival time, and the principal relaxation time, for random and ordered arrays of cylindrical fibers of various orientation distributions. The dimensionless mean survival time, principal relaxation time, mean pore size, and mean square pore size are found to increase with porosity, remain practically independent of the directionality of random fiber beds, and attain lower values for ordered arrays. Wide pore size distributions are obtained for random fiber structures and relatively narrow for ordered square arrays, all in very good agreement with theoretically predicted limiting values. Analytical results derived for the pore size probability and its lower moments for square arrays of fibers practically coincide with the corresponding simulation results. Earlier variational bounds on the mean survival time and principal relaxation time are obeyed by our numerical results in all cases, and are found to be quite sharp up to very high porosities. Dimensionless groups representing the deviation of such bounds from our simulation results vary in practically the same range as the corresponding values reported earlier for beds of spherical particles. A universal scaling expression of the literature relating the mean survival time to the mean pore size [S. Torquato and C. L. Y. Yeong, J. Chem. Phys. 106, 8814 (1997)] agrees very well with our results for all types of fiber structures, thus validated for the first time for anisotropic porous media. (C) 2003 American Institute of Physics. [DOI: 10.1063/1.1582431]
\end{abstract}

\section{INTRODUCTION}

Fibrous porous media are encountered in a broad spectrum of modern technology applications, including paper products, thermal insulations, fibrous filters and membranes, physiological systems and processes, and fiber-reinforced composites used extensively in the aerospace and automobile industry. In the recent past, Tomadakis and Sotirchos ${ }^{1-3}$ investigated the formation factor of random fibrous structures of various orientation distributions, allowing prediction of the corresponding thermal and electrical conductivities, dielectric constants, molecular diffusivities and magnetic permeabilities; they further examined the fiber structures with regards to their percolation and diffusion properties, as well as the track length distributions resulting from their traversal by random lines. ${ }^{4}$ Pore size distribution functions have been reported by other researchers for two-dimensional arrays of fibers, i.e., arrays of random lines on a plane. ${ }^{5}$

Experimental and theoretical investigations by Sotirchos and co-workers ${ }^{6}$ revealed the strong influence of pore size distribution on the transport of gas mixtures in porous media and the performance of gas-solid reaction processes, such as the desulfurization of coal gas using limestone or zinc oxide sorbent particles. Prager ${ }^{7}$ used the pore size distribution function to derive variational bounds and estimates of interphase transfer rates in random two-phase media. Torquato and co-workers ${ }^{8-10}$ used the function in studies of diffusion and reaction, $\mathrm{x}$-ray tomographic analysis, and digitization and numerical reconstruction of porous media, such as sandstones, beds of spheres, and porous gels.

A number of studies on porous media ${ }^{9-14}$ involve the concept of mean survival time, i.e., the average travel time of a molecule in steady-state Brownian diffusion before colliding and reacting with the pore wall, under infinite surface reaction rate conditions. Some of these studies also involve the broader concept of survival probability, representing the fraction of molecules that survive (do not react) up to a certain time during the process; the survival probability has also been the focus of extensive research on diffusion-controlled reactions in nonporous environments. ${ }^{15}$ The notion of mean survival time is equivalent to that of a mean residence time in a portion of the coordinate space, ${ }^{16}$ and is integral to many diffusion and reaction processes involving porous media. For instance, Drewry and Seaton ${ }^{12}$ used their simulation results for the mean survival time to derive the rate constant for heterogeneous reaction in catalyst particles. Torquato and $\mathrm{Kim}^{13}$ presented a direct link between the survival probability and the volume-integrated magnetization, a quantity of principal interest in nuclear magnetic resonance (NMR) studies. Torquato and Yeong ${ }^{14}$ derived a universal scaling relation linking the mean survival time to the mean pore size, porosity, and specific surface area of the porous medium.

Banavar and Schwartz ${ }^{17}$ applied numerical calculations 
on two types of granular media, in search of a correlation between the viscous permeability $k$ and the NMR relaxation time, $T_{1}$; they found a strong correlation between $k$ and the group $\varepsilon^{4} T_{1}^{2}, \varepsilon$ being the porosity, for fully saturated systems. This relation was later tested and validated or moderately upgraded for various types of porous media, such as beds of spherical particles, polymer membranes, and consolidated sandstones. ${ }^{18,19}$ Borgia et al. ${ }^{19}$ discussed a number of studies on this subject; they also presented their own experimental investigation of the relationship between $k$ and $T_{1}, \varepsilon, F(F$ being the formation factor), and concluded that the relaxation time is the decisive parameter for the estimation of the viscous permeability $k$. Torquato and co-workers ${ }^{13,20}$ developed a series of variational bounds and approximations relating the viscous permeability of porous media to the mean survival time, principal relaxation time, and other diffusion parameters. Torquato and Avellaneda ${ }^{10}$ derived lower bounds on the principal relaxation time $T_{1}$ and mean survival time $\tau$, in terms of the moments of the pore size distribution, and evaluated these moments and bounds for random arrays of overlapping and nonoverlapping spheres.

The purpose of our present investigation is to compute the pore size probability distribution and its moments, the survival probability and mean survival time, and the principal relaxation time, for various types of fiber structures consisting of cylindrical fibers with axes parallel to a line (1d random or ordered in a square array), parallel to a plane (2d random), or oriented randomly in the three-dimensional space ( $3 \mathrm{~d}$ random). In Sec. II, we outline the basic theoretical principles and methods involved in this study. In Sec. III, we present our numerical results for all types of investigated fiber structures, along with our analytical results for the $1 \mathrm{~d}$ square array, and compare them to variational bounds, earlier results on arrays of spheres, and the above mentioned universal scaling relation. Our main conclusions are summarized in Sec. IV.

\section{THEORETICAL PRINCIPLES AND METHODS}

\section{A. Pore size distribution}

Scheidegger ${ }^{20}$ defined the "pore diameter" $\delta$ at any point within the pore space as "the diameter of the largest sphere which contains this point and remains wholly within the pore space." He further defined the pore size distribution "based on the fraction $P(\delta)$ of the total pore space that has a pore diameter between $\delta$ and $\delta+d \delta$." $P(\delta)$ is normalized by the well known equation

$$
\int_{0}^{\infty} P(\delta) d \delta=1
$$

with the corresponding cumulative pore size distribution, $F(\delta)$, given by

$$
F(\delta)=\int_{\delta}^{\infty} P(\delta) d \delta .
$$

Prager $^{7}$ interpreted Scheidegger's definition of $\delta$ as the size of the largest sphere centered at the point, i.e., he in effect redefined $\delta$ as the minimum distance of the point from the pore surface. By Prager's definition, the pore size distribu- tion probability $P(\delta) d \delta$ is the probability that a randomly chosen point in the pore region lies at a distance between $\delta$ and $\delta+d \delta$ from the nearest point on the pore-solid interface. This definition facilitates the numerical computation of parameter $\delta$ and the corresponding pore size distribution, and was adopted in a number of later studies $;{ }^{8-10}$ nevertheless, it should be born in mind that this modified $\delta$ is not any more a pore diameter, but rather a much smaller characteristic pore size, with values ranging from 0 to the pore radius, $r_{p}$. For instance, the mean pore size of an infinitely long cylindrical pore of radius $r_{p}$ is readily derived [from $\langle\delta\rangle$ $=(1 / V) \int \delta d V, V$ being the pore volume] as $\left\langle\delta_{c}\right\rangle=r_{p} / 3$, while that of a spherical pore as $\left\langle\delta_{s}\right\rangle=r_{p} / 4$.

The pore size distribution probability is of course independent of the employed unit length, therefore $P(\delta) d \delta$ $=P(\delta / r) d(\delta / r)$ and $P(\delta / r)=r P(\delta)$. At the extreme values of $\delta, P(\delta / r)$ attains the values

$$
P(0)=\frac{S}{V}=\frac{S_{b}}{\varepsilon}=\frac{4}{\bar{d}},
$$

and

$$
P(\infty)=0,
$$

where $S$ and $S_{b}$ are the surface area and specific surface area of the porous medium, respectively, $\varepsilon$ is the porosity, and $\bar{d}$ the mean intercept length. For randomly overlapping fiber structures, $\bar{d}$ is given from ${ }^{1}$

$$
\bar{d}=2 \bar{r}=\frac{-2 r}{\ln \varepsilon},
$$

where $r$ is the fiber radius and $\bar{r}$ is the mean intercept halflength (an average pore radius). For random nonoverlapping fiber structures, such as the square array studied here, the mean intercept length is obtained from ${ }^{2}$

$$
\bar{d}=2 \bar{r}=\frac{2 \varepsilon r}{1-\varepsilon} .
$$

Substituting these expressions into Eq. (3a), we obtain for the random arrays,

$$
P(0)=-2 \ln \varepsilon,
$$

and for the square array

$$
P(0)=2\left(\frac{1}{\varepsilon}-1\right) \text {. }
$$

The moments of the pore size distribution $P(\delta)$ are defined from

$$
\left\langle\delta^{n}\right\rangle=\int_{0}^{\infty} \delta^{n} P(\delta) d \delta .
$$

The lower-order moments $\langle\delta\rangle$ and $\left\langle\delta^{2}\right\rangle$ can be used to derive lower bounds on the mean survival time, $\tau$, and the principal relaxation time, $T_{1}$, as discussed in the following section.

\section{B. Survival and relaxation time}

The mean survival time, $\tau$, of molecules undergoing steady-state Brownian diffusion may be obtained from integration of the corresponding survival probability, $S(t)$, 


$$
\tau=\int_{0}^{\infty} S(t) d t .
$$

Torquato and $\mathrm{Kim}^{13}$ expressed the survival probability as an infinite summation involving the diffusion relaxation times, $T_{n}$, associated with the time-dependent solution of the Laplace equation for diffusional flow; they showed that for large $t$,

$$
\ln S(t) \sim t / T_{1}
$$

where $T_{1}$ is the principal or largest diffusion relaxation time, which may thence be obtained from the inverse of the slope of $\ln S(t)$ versus $t$, for large values of the survival time, $t$.

Prager $^{7}$ used the lower-order moments $\langle\delta\rangle$ and $\left\langle\delta^{2}\right\rangle$ of the pore size distribution function $P(\delta)$ to obtain rigorous lower bounds on $\tau$ and $T_{1}$ for diffusion-controlled reactions (infinite surface reaction rates),

$$
\begin{gathered}
\tau \geqslant \frac{\langle\delta\rangle^{2}}{D}, \\
T_{1} \geqslant \frac{\left\langle\delta^{2}\right\rangle}{D} .
\end{gathered}
$$

Torquato and Avellaneda ${ }^{10}$ derived generalized versions of these bounds accounting for finite surface reaction rates. They claimed that Eq. (12) should provide a rather coarse estimate of $T_{1}$ for systems of very wide fluctuations in pore size, while Eq. (11) should be a more robust estimator of $\tau$, especially at low porosities.

Although most relevant literature studies refer to the survival time of molecules in a porous medium, the truly structure-dependent parameter is the dimensionless survival distance (total path length until molecule reacts), $s / r$. Once the average value $\bar{s} / r$ of this parameter is obtained, the corresponding mean survival time $\tau$ may be readily estimated from $\tau=\bar{s} / \bar{v}$, where $\bar{v}$ is the molecular mean thermal speed. The resulting value of $\tau$ may be rendered dimensionless by means of Eqs. (4) or (5) and the fundamental relations $D$ $=1 / 3 \lambda \bar{v}$ and $\mathrm{Kn}=\lambda / \bar{d}$, relating the no-memory Brownian random walk diffusion coefficient $(D)$ and the Knudsen number $(\mathrm{Kn})$ to the molecular mean free path $(\lambda)$, mean thermal speed $(\bar{v})$, and mean intercept length $(\bar{d})$. The resulting dimensionless mean survival time is given for randomly overlapping fiber structures by

$$
\frac{\tau D}{r^{2}}=\frac{-2 \operatorname{Kn}(\bar{s} / r)}{3 \ln \varepsilon}
$$

and for unidirectional nonoverlapping fibers, such as the $1 \mathrm{~d}$ square array, by

$$
\frac{\tau D}{r^{2}}=\frac{2 \varepsilon \operatorname{Kn}(\bar{s} / r)}{3(1-\varepsilon)} .
$$

The dimensionless mean survival distance $\bar{s} / r$ may be obtained from random walk simulations using $\mathrm{Kn}=0.02$, i.e., in the bulk regime, where Brownian diffusion prevails.

\section{Universal scaling}

Torquato and Yeong ${ }^{14}$ used rigorous bounds on the mean survival time, along with their own and earlier theoretical results on $\tau$ and $\langle\delta\rangle$ for various random and ordered isotropic arrays of spheres and digitized media of $\varepsilon \leqslant 0.9$, to derive the following universal scaling relation for diffusion-controlled reactions in porous media:

$$
\frac{\tau}{\tau_{0}}=\frac{8}{5} \chi+\frac{8}{7} \chi^{2},
$$

where the reference time $\tau_{0}$ and the length scale $\chi$ are given from

$$
\begin{aligned}
& \tau_{0}=\frac{3(1-\varepsilon)}{D \varepsilon S_{b}^{2}}, \\
& \chi=\frac{\langle\delta\rangle^{2}}{\tau_{0} D} .
\end{aligned}
$$

According to Eqs. (15)-(17), the mean survival time, $\tau$, may be predicted from the values of porosity, $\varepsilon$, specific surface area, $S_{b}$, mean pore size, $\langle\delta\rangle$, and bulk diffusion coefficient, $D$. Torquato and Yeong ${ }^{14}$ claim that this relation should be applicable to a wide class of microstructures provided that $\chi<0.5$, as was the case with the data employed in its derivation.

\section{Brownian diffusion random walk}

The survival probability and mean survival time are estimated in this study from an earlier Brownian diffusion random walk simulation algorithm, ${ }^{1}$ marginally modified to account for molecular trajectory termination at first impact with the solid surface. A unit cubic cell is created first to represent numerically the random fiber structure; this is equivalent to the problem of generating a random line population in a convex body. Coleman ${ }^{22}$ described various ways such random secants could arise, with "mean free path randomness," or " $\mu$-randomness," being the mechanism producing statistically homogeneous structures: The secants are defined by a random point on a reference plane and a random direction with respect to that plane, and have size and orientation distributions identical to those of actual paths of molecules moving randomly in the empty cell (i.e., reflecting on the six faces of the cube).

Trajectory computations begin by generating a random position within the unit cell. If the point falls within the void space it becomes the starting position for a random walk. A random direction is assigned to the walker, and a molecular free path $\lambda$ is sampled from an exponential distribution. Before the molecule can be moved to this point, verification must be made that the molecule will not encounter a fiber or cell boundary within this distance. If no interference is found, the molecule is advanced the distance $\lambda$. If one or more fibers lie within the path of the advancing molecule, the shortest distance between the point of origin for this step and the fibers determines the next position of the walker. When infinite surface reaction rate is considered, no reflection of 


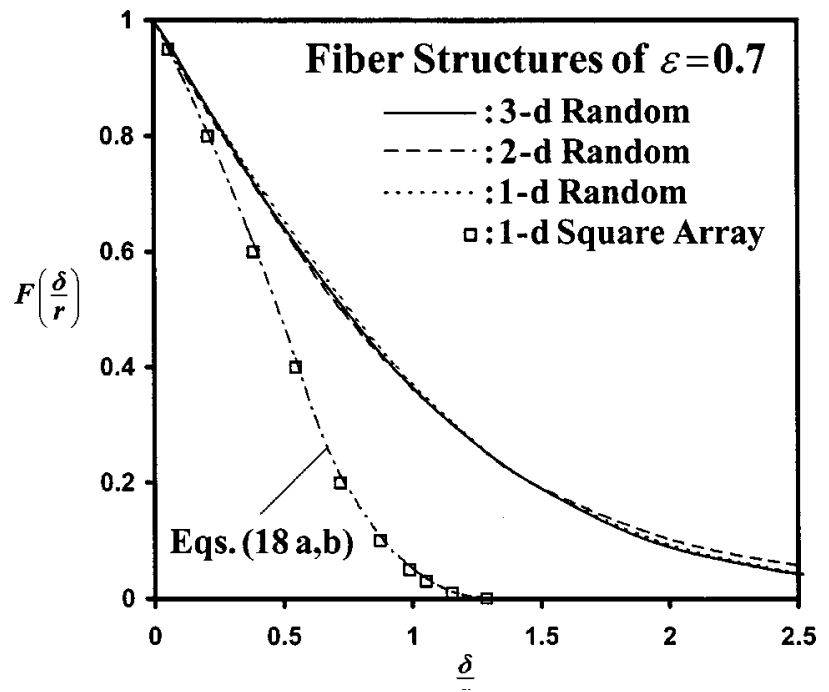

FIG. 1. Cumulative pore size probability distribution of random and ordered fiber structures of various orientation distributions.

the walker from the solid surface is accounted for, and the simulation continues by placing another random walker in the unit cell.

When a random walker encounters the unit cell boundary, it is specularly reflected on it and reintroduced to the unit cell. This is equivalent to assuming that the porous medium is an infinite assemblage of identical unit cells where each neighboring cell is a mirror image of the next. The use of such boundary conditions does not introduce any measurable bias in the simulation results, provided that a high enough population of fibers is present in the unit cell, i.e., a big enough sample of the random fiber structure is investigated. ${ }^{23}$ The computation of the molecular trajectories is accelerated considerably by using time-saving modifications of the basic simulation algorithm, namely, a spherical inclusion technique exploiting the basic idea of first-passagetime (FPT) random walks, and certain domain and path discretization schemes. ${ }^{23}$

By keeping track of the travel distance of the random walkers, as well as their initial distance from the closest solid surface, survival probability and pore size distributions can be derived for all types of random and ordered fiber structures covering a broad range of porosities.

\section{RESULTS AND DISCUSSION}

\section{A. Pore size distribution}

Figure 1 presents the cumulative probability distribution $F(\delta / r)$ at $\varepsilon=0.7$ for all four types of random and ordered fiber structures investigated in this work. As mentioned in Sec. II A, $F(\delta / r)$ represents the fraction of points in the porous space whose dimensionless distance from the solid surface is higher than $\delta / r$; naturally, all cumulative pore size distribution curves originate from point $(0,1)$. No significant fiber directionality effect is encountered for the three random structures, since the $1 \mathrm{~d}, 2 \mathrm{~d}$, and $3 \mathrm{~d}$ curves practically coincide; this was found to be the case throughout the porosity range. As shown in Fig. 1, the random beds encounter a wider range of pore sizes than the ordered square array, since

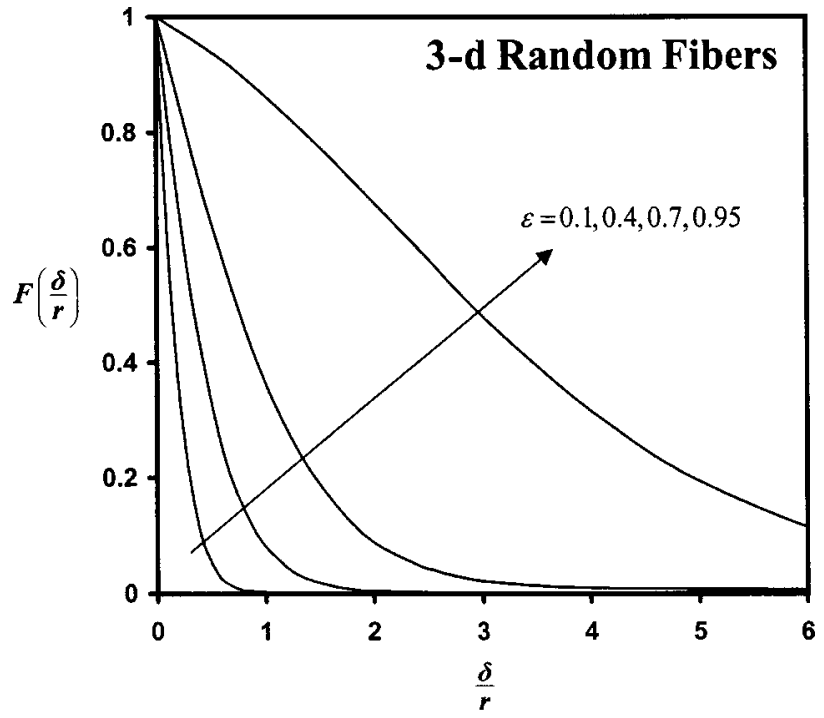

FIG. 2. Cumulative pore size probability distribution of three-directional random fiber structures of various porosities.

their stochastic nature allows for both very small and very big voids. The ordered nature of the square array facilitates geometrical derivation of analytical expressions for the cumulative pore size probability distribution. Setting $\xi=1$ $+\delta / r$ and $\eta=r_{\mathrm{cp}} / r=\frac{1}{2} \sqrt{\pi /(1-\varepsilon)}$, where $r_{\mathrm{cp}}$ is the close packing radius of the structure, we obtain

$$
\begin{aligned}
& \text { For } \xi \leqslant \eta: F(\xi)=1-\left(1-\varepsilon^{-1}\right)\left(1-\xi^{2}\right), \\
& \text { For } \xi \geqslant \eta: F(\xi) \\
& \qquad \begin{aligned}
\eta^{2}+\xi^{2}\left[\cos ^{-1}(\eta / \xi)-\pi / 4\right]-\eta \sqrt{\xi^{2}-\eta^{2}} \\
\eta^{2}-\pi / 4
\end{aligned}
\end{aligned}
$$

The maximum value of $\xi$ may be obtained from simple geometry as $\xi_{\max }=\eta \sqrt{2}$, predicted also by Eq. (18b) for $F(\xi)$ $=0$. For $\xi=\eta$, Eqs. (18a) and (18b) reduce to the trivial $F(\xi)=\varepsilon_{\mathrm{cp}} / \varepsilon$, where $\varepsilon_{\mathrm{cp}}=1-\pi / 4$ is the close packing porosity of the square array. As shown in Fig. 1, both equations are in very good agreement with our simulation results for the square array of fibers.

The porosity effect on the cumulative pore size distribution of a $3 \mathrm{~d}$ random fiber structure is illustrated in Fig. 2; it can be seen that higher-porosity beds encounter higher values of $\delta / r$ due to the bigger voids they contain, hence they also exhibit higher $F(\delta / r)$ values at any given $\delta / r$. Figure 3 presents the pore size distribution $P(\delta / r)$ at $\varepsilon=0.4$ for all types of random and ordered fiber structures under consideration. Since $P(\delta / r)$ represents the derivative of $F(\delta / r)$ at any given point, its values were derived from the cumulative distribution using a numerical differentiation scheme. For all curves shown in Fig. 3, the pore size probability density $P(0)$ agrees very well with the earlier discussed theoretical predictions [Eqs. (6) and (7)]. Furthermore, all differences in the $P(\delta / r)$ values of the three random array curves are of the order of their differences at $\delta / r=0$, which are known from Eq. (6) to be due to their minor porosity deviations from $\varepsilon=0.4( \pm 0.005)$. This is true also for all other porosities investigated in this study, which leads us to postulate that all 


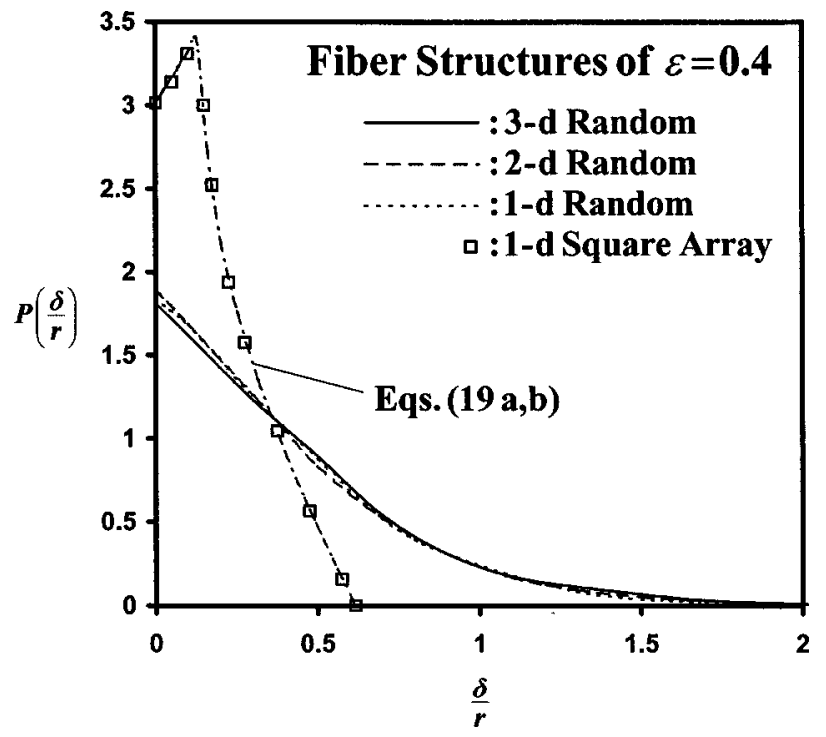

FIG. 3. Pore size probability distribution of random and ordered fiber structures of various orientation distributions.

$1 \mathrm{~d}, 2 \mathrm{~d}$, and $3 \mathrm{~d}$ random beds of the same porosity exhibit practically identical pore size distribution. As also deduced from Fig. 3, the maximum pore size probability density, $P(\delta / r)_{\max }$, attains a higher value for the square array structures, due to the narrower pore size distributions encountered in such beds.

Analytical predictions for the pore size distribution of square arrays of fibers may be readily obtained through differentiation of Eqs. (18a) and (18b),

$$
\begin{aligned}
& \text { For } \xi \leqslant \eta: P(\xi)=2 \xi\left(1-\varepsilon^{-1}\right), \\
& \text { For } \xi \geqslant \eta: P(\xi)=\frac{2 \xi\left[\cos ^{-1}(\eta / \xi)-\pi / 4\right]}{\eta^{2}-\pi / 4} .
\end{aligned}
$$

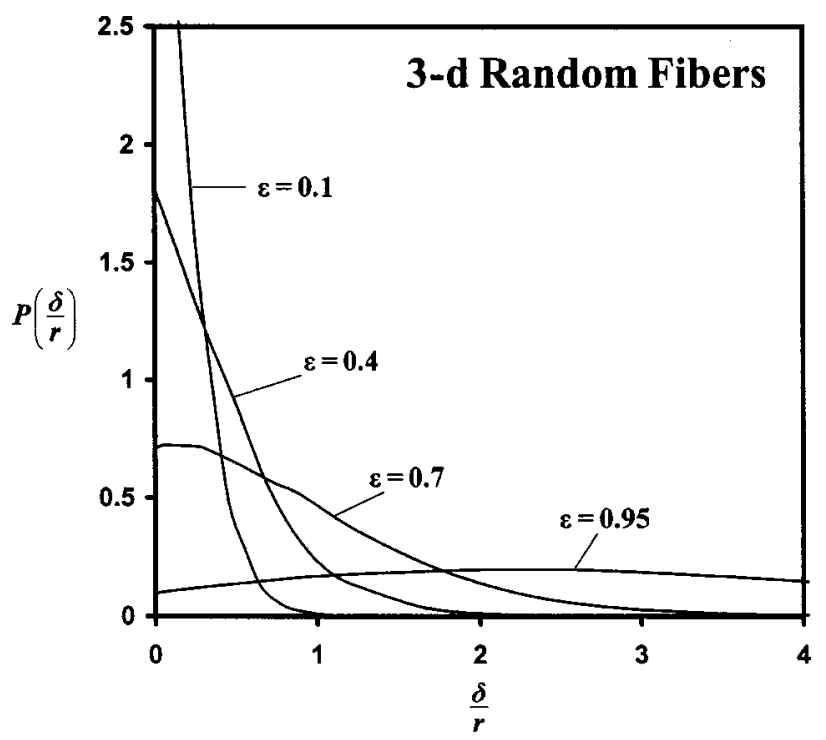

FIG. 4. Pore size probability distribution of three-directional random fiber structures of various porosities.

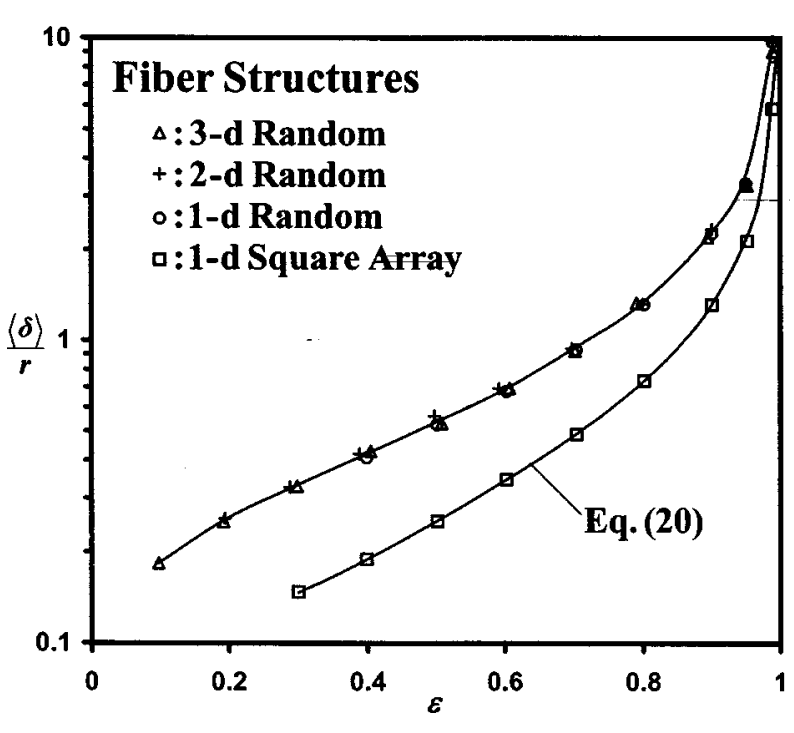

FIG. 5. Variation of the dimensionless mean pore size [first moment of $P(\delta)$ ] with the porosity for random and ordered fiber structures of various orientation distributions.

These equations are plotted in Fig. 3 for the square array of $\varepsilon=0.4$, where they are seen to agree very well with our simulation results for that fiber structure.

Figure 4 shows the $P(\delta / r)$ curves for $3 \mathrm{~d}$ random structures of various porosities, derived from the cumulative probabilities $F(\delta / r)$ shown in Fig. 2. Our numerical estimates for $P(0)$ agree very well with the predictions of Eqs. (6) and (7), as was the case with the results presented in Fig. 3. Furthermore, lower values of $P(\delta / r)_{\max }$ are encountered at higher porosities, due to the wider pore size distributions in dilute beds; naturally, the location of $P(\delta / r)_{\max }$ shifts to higher $\delta / r$ values as the porosity increases, due to the presence of bigger voids in such beds.

David $^{24}$ employed numerical simulations on twodimensional ordered pore networks to show that when the pore size distribution has a decreasing exponential-like shape - as opposed to nearly uniform distributions - the "hydraulic tortuosity" of the porous medium is much higher than its "electrical tortuosity," by a factor of about 4 on the average for the networks he studied. Since the pore size distributions computed in our study for random fiber structures are indeed of that form, David's observation is in excellent agreement with our own recent results on the relative value of the viscous and bulk tortuosity of random beds of fibers. ${ }^{25}$

Figures 5 and 6 present the variation of the dimensionless first and second moments of $P(\delta / r)$, namely, the mean pore size, $\langle\delta\rangle / r$, and the mean square pore size, $\left\langle\delta^{2}\right\rangle / r^{2}$, with the porosity, for all four types of fiber structures examined in our study. In accordance with the observations made in Figs. 1-4, the random bed values of both moments are independent of fiber directionality and considerably higher than the corresponding square array values, at any porosity level. The latter are in excellent agreement with the analytical predictions obtained from Eqs. (8), (19a), and (19b),

$$
\frac{\left\langle\delta_{\mathrm{sq}}\right\rangle}{r}=\frac{[\sqrt{2}+\ln (\sqrt{2}+1)] \eta-\varepsilon-2}{3 \varepsilon},
$$




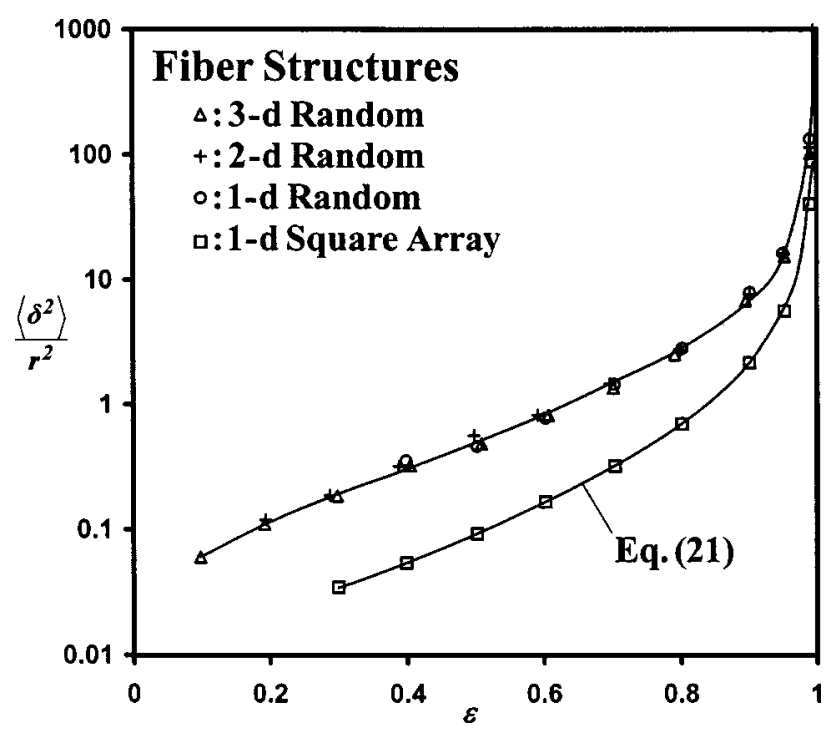

FIG. 6. Variation of the dimensionless mean square pore size [second moment of $P(\delta)]$ with the porosity for random and ordered fiber structures of various orientation distributions.

$$
\frac{\left\langle\delta_{\mathrm{sq}}^{2}\right\rangle}{r^{2}}=\frac{4 \eta[\eta-\sqrt{2}-\ln (\sqrt{2}+1)]+\varepsilon+5}{6 \varepsilon} .
$$

A regression analysis of the ratio of simulation estimate to analytical prediction for square arrays of $\varepsilon \leqslant 0.9$ gives $\left\langle\langle\delta\rangle_{\text {sim }} /\langle\delta\rangle_{\text {Eq. (20) }}\right\rangle=1.001 \pm 0.003$ and $\left\langle\left\langle\delta^{2}\right\rangle_{\text {sim }} /\right.$ $\left.\left\langle\delta^{2}\right\rangle_{\text {Eq. (21) }}\right\rangle=1.000 \pm 0.007$, underlining the nearly perfect agreement of numerical and analytical results. For the randomly overlapping fiber structures, the ratio $\left\langle\delta^{2}\right\rangle /\langle\delta\rangle^{2}$ varies from 1.79 to 1.39 in the porosity range $0.1-0.9$, in very good agreement with the observations of Torquato and Avellaneda ${ }^{10}$ for beds of randomly overlapping spheres, where the same ratio is reported to vary from 1.73 to 1.35 , over the same range of porosity.

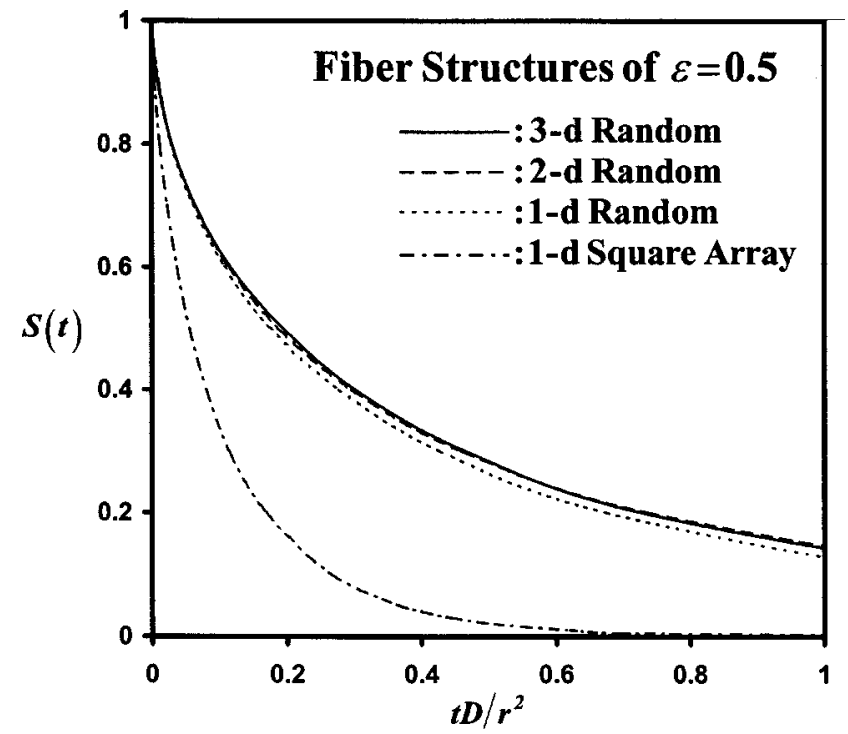

FIG. 7. Survival probability distribution in random and ordered fiber structures of various orientation distributions.

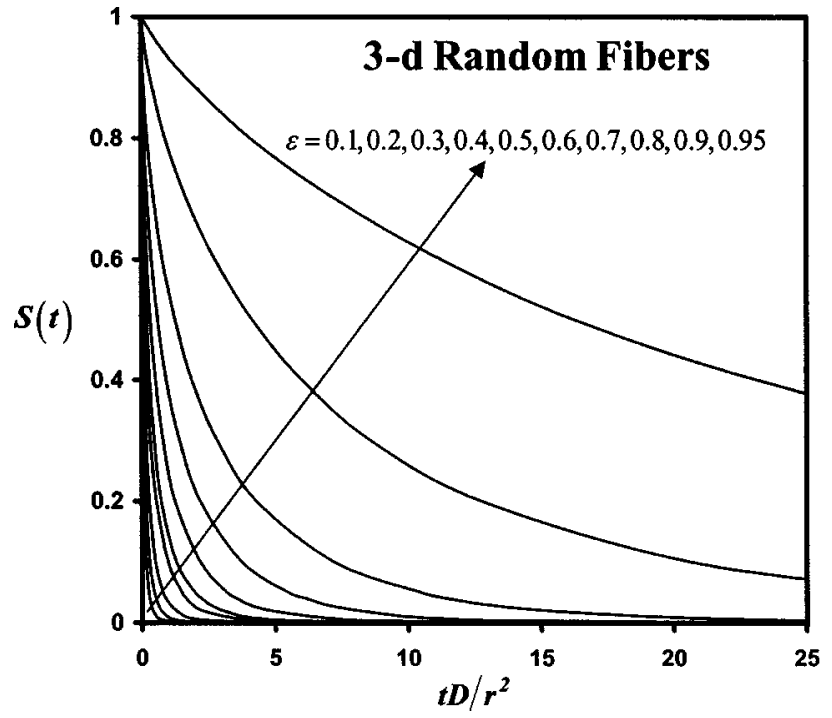

FIG. 8. Survival probability distribution in three-directional random fiber structures of various porosities.

\section{B. Survival probability}

Figure 7 presents the survival probability distribution $S(t)$ at $\varepsilon=0.5$ for all random and ordered arrays of fibers investigated in our work, with each curve obtained from 10000 random walks. As shown in the figure, the three random structures behave practically as one, as was the case with the corresponding pore size distributions presented in Sec. III A. The ordered array encounters lower $S(t)$ values, since molecules terminate their trajectories sooner due to the smaller voids contained in such structures. A more elaborate look at the survival probability is offered in Figs. 8 and 9, which show the evolution of $S(t)$ with $\varepsilon$ throughout the porosity range for the $3 \mathrm{~d}$ random and $1 \mathrm{~d}$ square array, respectively. Both figures show higher survival probability values at higher porosities, as anticipated by intuition, with the difference becoming more pronounced for very dilute beds.

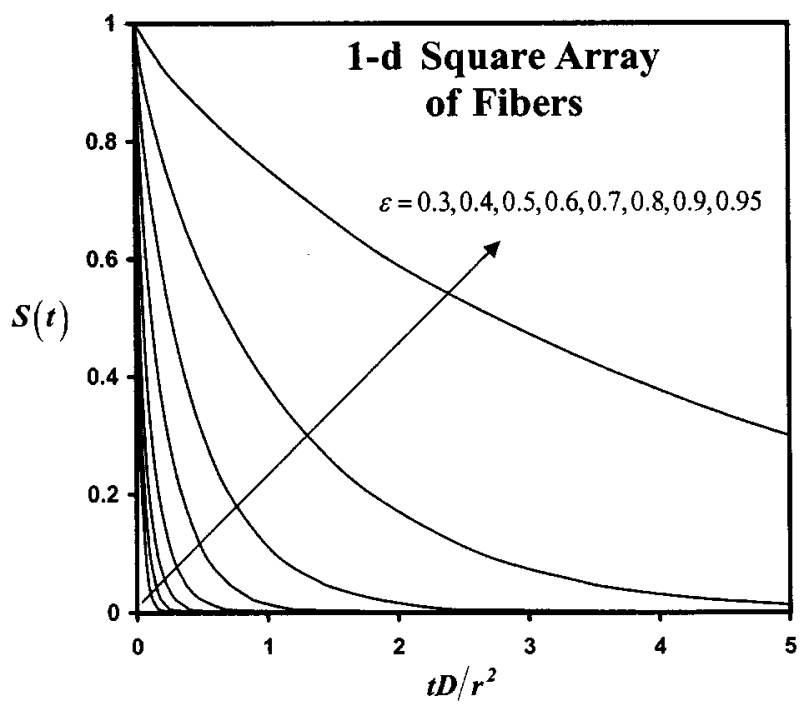

FIG. 9. Survival probability distribution in unidirectional square arrays of fibers of various porosities. 


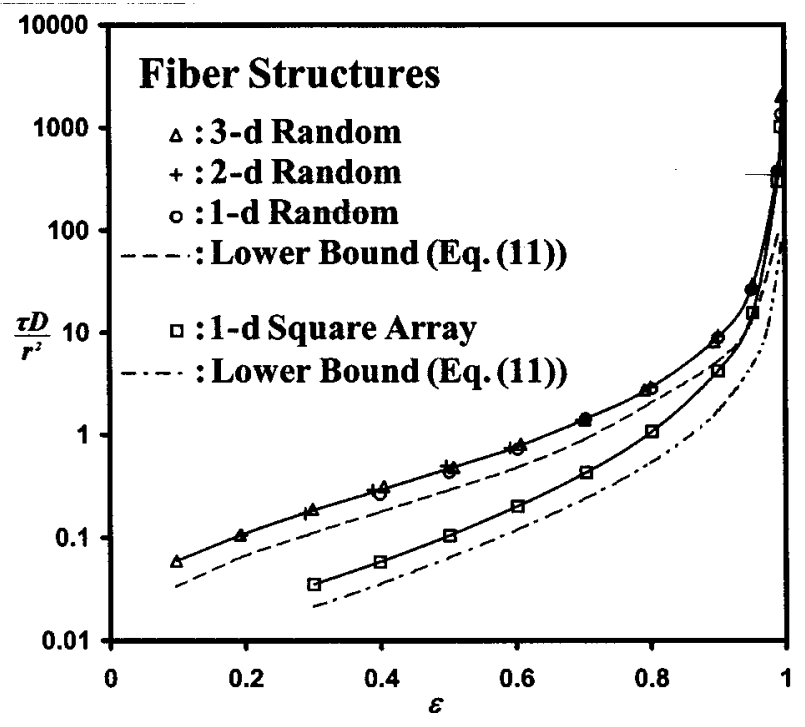

FIG. 10. Variation of the dimensionless mean survival time with the porosity for random and ordered fiber structures of various orientation distributions.

The variation of the dimensionless mean survival time, $\tau D / r^{2}$, with the porosity is presented in Fig. 10. In agreement with the observations made in Fig. 7, minor differences are encountered in the behavior of $1 \mathrm{~d}, 2 \mathrm{~d}$, and $3 \mathrm{~d}$ random beds of fibers, but considerably lower survival times are obtained for square arrays. The lower bound by Prager $^{7}$ and Torquato and Avellaneda ${ }^{10}$ [Eq. (11)] is also plotted in Fig. 10 for both the random and ordered fiber structures, as derived from the $\langle\delta\rangle / r$ values presented in Fig. 5. It is readily seen that the bound is quite sharp, and is obeyed by our simulation results for all structures, throughout the porosity range. For $\varepsilon \leqslant 0.9$, the bound underpredicts the simulation results by a factor of 1.5-1.8 for random arrays and 1.6-2.4 for square arrays, with the deviation becoming more pronounced at higher porosities. Torquato and Yeong ${ }^{14}$ plot $\tau D / r^{2}$ versus $\langle\delta\rangle^{2} / r^{2}$ for five types of random and ordered, overlapping and nonoverlapping arrays of spheres of $\varepsilon \leqslant 0.9$. The ratios $\tau /\left(\langle\delta\rangle^{2} / D\right)$ obtained from their data vary from 1.75 to 2.1 , in very good agreement with our results for fiber structures.

Table I offers a comparison of the dimensionless mean survival distance, $\bar{s} / r$, to the mean intercept radius, $\bar{r} / r$, and

TABLE I. Comparison of the mean survival distance to the mean pore size and mean intercept radius for $3 \mathrm{~d}$ random arrays of fibers.

\begin{tabular}{ccccc}
\hline \hline$\varepsilon$ & $\bar{s} / r$ & $\bar{r} / r$ & $\langle\delta\rangle / r$ & $\bar{r} /\langle\delta\rangle$ \\
\hline 0.99 & 285.9 & 99.5 & 9.02 & 11.0 \\
0.95 & 108.1 & 20.8 & 3.27 & 6.35 \\
0.9 & 67.6 & 9.01 & 2.20 & 4.10 \\
0.8 & 48.1 & 4.27 & 1.33 & 3.21 \\
0.7 & 37.1 & 2.83 & 0.92 & 3.07 \\
0.6 & 30.2 & 2.00 & 0.69 & 2.90 \\
0.5 & 24.5 & 1.48 & 0.53 & 2.79 \\
0.4 & 21.3 & 1.11 & 0.43 & 2.57 \\
0.3 & 17.1 & 0.83 & 0.33 & 2.51 \\
0.2 & 13.1 & 0.61 & 0.25 & 2.42 \\
0.1 & 10.3 & 0.43 & 0.18 & 2.39 \\
\hline \hline
\end{tabular}

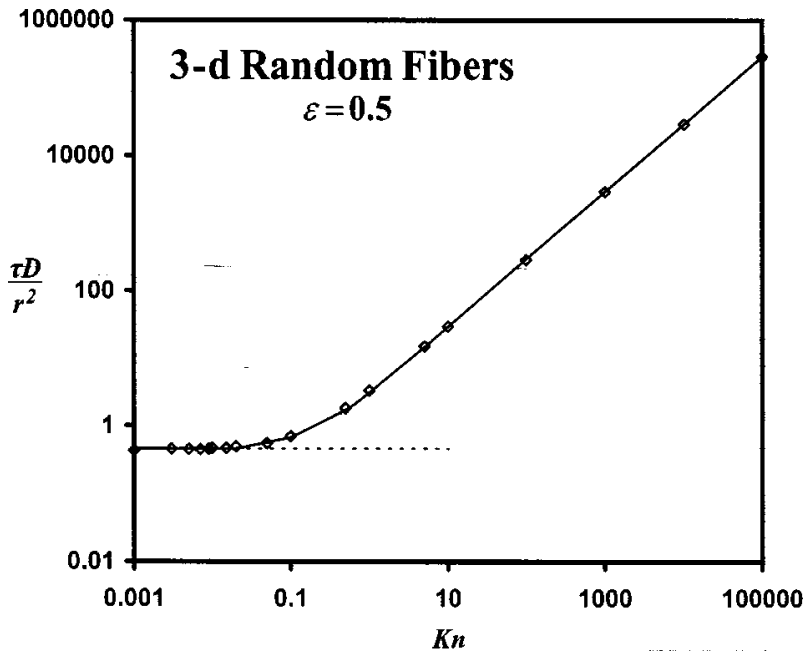

FIG. 11. Variation of the dimensionless mean survival time with the Knudsen number.

the mean pore size, $\langle\delta\rangle / r$, for $3 \mathrm{~d}$ random fiber beds of various porosities. As is evident on this table, the mean survival distance is much higher than both other values, as it represents the total length of a multistep Brownian movement from the void space to the fiber surface. On the other hand, the mean pore size $\langle\delta\rangle / r$ represents the one-step shortest distance from a point in the void space to the solid surface, therefore it attains the lowest values among all three parameters. The dimensionless mean intercept radius $\bar{r} / r$ represents also a one-step distance from the void space to the fiber surface; however, instead of the shortest distance, intercept steps follow the $\mu$-random orientation distribution of actual molecular paths, which makes their average size higher than $\langle\delta\rangle / r$. Table I shows also the relative values $\bar{r} /\langle\delta\rangle$ in $3 \mathrm{~d}$ random fiber structures; interestingly, for the most part of the porosity range, this ratio varies from about 2.4 to 3.2 , i.e., very close to the values $2.67(=8 / 3)$ and 3 corresponding to spherical and cylindrical pores, respectively (derived from $\bar{r}=2 \mathrm{~V} / \mathrm{S}$ and the values of $\langle\delta\rangle / r$ presented in Sec. II A). This is not the case with very dilute beds, where the fibers occupy a very small part of the total volume, hence $\bar{r}$ increases dramatically with $\varepsilon$ while $\langle\delta\rangle$ increases much slower, as it represents the distance from the closest fiber, not one hit randomly.

All survival time and distance results presented as distributions or mean values in Figs. 7-10 and Table I were obtained from Brownian diffusion random walk simulations, using a Knudsen number value $\mathrm{Kn}=0.02$. Figure 11 shows the dependence of such numerical results on $\mathrm{Kn}$ for a $3 \mathrm{~d}$ random bed of fibers of $\varepsilon=0.5$. As shown on this plot, the dimensionless mean survival time $\tau D / r^{2}$ is practically independent of the Knudsen number for $\mathrm{Kn} \leqslant 0.02$, i.e., as long as the random walks are carried out in the Brownian diffusion regime. For high values of $\mathrm{Kn}$, one straight path step is all it takes for a molecule to reach the solid surface, which transforms the mean survival distance to a mean intercept radius of fixed value, independent of $\mathrm{Kn}$. In accordance with Eq. (13), this results to the linear behavior of $\tau D / r^{2}$ versus $\mathrm{Kn}$ observed in Fig. 11. 


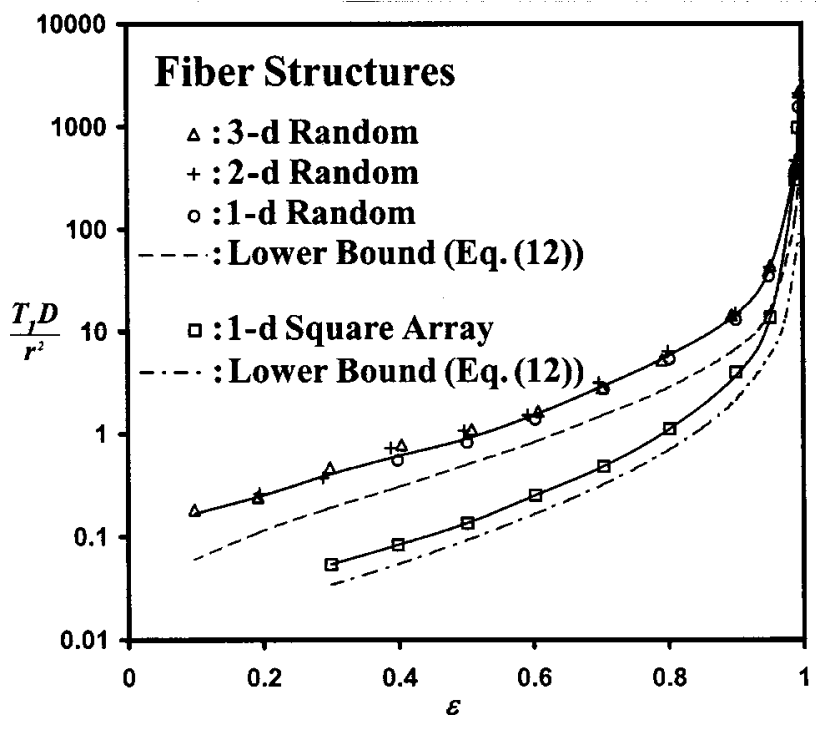

FIG. 12. Variation of the dimensionless principal relaxation time with the porosity for random and ordered fiber structures of various orientation distributions.

\section{Relaxation time}

Figure 12 presents the variation of the dimensionless principal relaxation time, $T_{1}$, with the porosity, for all fiber structures examined in this study. The results are qualitatively similar to those shown in Fig. 10 for the dimensionless mean survival time. The lower bound by Prager $^{7}$ and Torquato and Avellaneda ${ }^{10}$ [Eq. (12)], derived from our $\left\langle\delta^{2}\right\rangle / r^{2}$ data shown in Fig. 6 , is obeyed by our $T_{1}$ results in all cases; it is also less sharp for the random beds than the corresponding bound on $\tau$ (Fig. 10), in accordance with earlier predictions. ${ }^{10}$ The bound underpredicts the measured $T_{1}$ by a factor of about 2 for random beds and 1.5 for square arrays, for porosities in the range $0.2-0.9$, and by a higher factor for more dilute beds. The fact that the bound is sharper for the square array than it is for the random beds agrees with an earlier proposition that this bound should yield a reasonable estimate on the relaxation time of porous media with finite pore size range. ${ }^{10}$

A comparison of the $T_{1}$ results of Fig. 12 to the $\tau$ results of Fig. 10 reveals that the mean survival time $\tau$ is bounded from above by the relaxation time $T_{1}$, as suggested earlier based on theoretical results on beds of spheres. ${ }^{10}$ Furthermore, the ratio $T_{1} / \tau$ is found lower than 2.5 for all but the lowest-porosity random fiber beds, approaching unity in the dilute limit for all types of random and ordered fiber structures investigated in our work; this is in excellent agreement with the results of Torquato and $\mathrm{Kim}^{13}$ for beds of spheres ordered in $\mathrm{SC}, \mathrm{FCC}$, and $\mathrm{BCC}$ arrangement. Lower ratios are obtained for the square array of fibers, reaching a maximum of about 1.5 near the close packing limit, where molecules are practically isolated in identical single pores with concave walls. It may be worth mentioning that practically the same value of $T_{1} / \tau$, namely $15 / \pi^{2}=1.52$, was obtained earlier for diffusion interior to isolated spherical pores. ${ }^{10}$

The values of $T_{1}$ shown in Fig. 12 were derived from the corresponding survival probability distributions $S(t)$ using

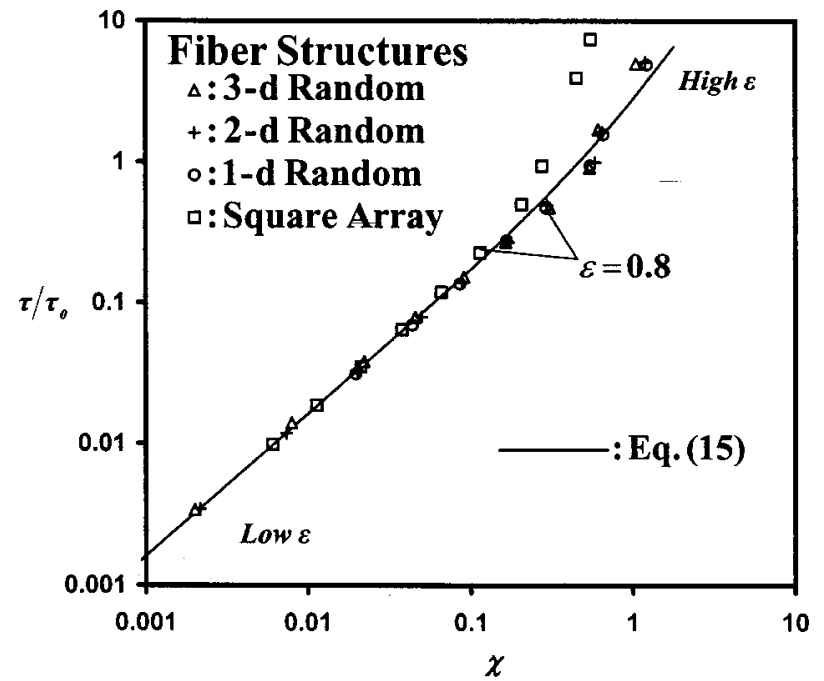

FIG. 13. Comparison of the universal scaling expression for the mean survival time [Eq. (15)] to our simulation results for random and ordered fiber structures of various orientation distributions.

Eq. (10); from the 10000 random walkers used to obtain $S(t)$, only the ten with the largest survival times (i.e., the top $0.1 \%$ ) were used to get the average slope of $\ln S(t)$ versus $t$, yielding $T_{1}$. All values of $T_{1}$ were then recalculated from only one slope value for each run (obtained from the two highest survival times), and compared to those presented in Fig. 12; a regression analysis gave $\left\langle T_{1}(10) / T_{1}(1)\right\rangle=0.98$ \pm 0.08 , with the average ratio and standard deviation being practically independent of porosity and type of structure. Eight more simulations were run for the $3 \mathrm{~d}$ random bed of $50 \%$ porosity, using 2500-65000 random walkers, and estimating $T_{1}$ again from the top $0.1 \%$ of the measured $t$ values. A regression analysis of the derived values gave $\left\langle T_{1}(N) / T_{1}\left(10^{4}\right)\right\rangle=1.01 \pm 0.05$, indicating again a negligible effect of the number of employed random walkers, $N$, on the accuracy of the estimated $T_{1}$.

\section{Universal scaling}

Our results for the mean survival time $\tau$ and the mean pore size $\langle\delta\rangle$ were scaled by means of the time scale $\tau / \tau_{0}$ and length scale $\chi$ using Eqs. (16) and (17), and plotted in Fig. 13 for comparison to the "universal curve" [Eq. (15)] proposed by Torquato and Yeong. ${ }^{14}$ The plot shows very good agreement of our results with Eq. (15) throughout the porosity range for all types of random fiber structures, and up to a very high porosity $(\varepsilon \approx 0.9)$ for the square array of fibers. This is the first time that Eq. (15) is validated for statistically anisotropic media, such as the $1 \mathrm{~d}$ square array and the $1 \mathrm{~d}$ and $2 \mathrm{~d}$ random fiber beds investigated in this work. This result lends further support to the universal character of this scaling expression which enables a direct estimation of any one of $\tau$, $\varepsilon, S_{b},\langle\delta\rangle$, from the values of the other three parameters.

\section{SUMMARY AND REMARKS}

We investigated the pore size probability distribution and its moments, the survival probability and mean survival time, 
and the principal relaxation time, for various types of fiber structures. We considered random porous media consisting of cylindrical fibers randomly distributed in space with their axes parallel to a line (1d), parallel to a plane (2d), or oriented randomly in the three-dimensional space (3d), with the fibers allowed to overlap freely in all three cases. We also studied an ordered structure, formed by parallel cylindrical nonoverlapping fibers arranged in a square-array configuration.

We applied a Brownian diffusion random-walk simulation technique to obtain the pore size distribution $P(\delta)$ and survival probability $S(t)$ in the randomly overlapping and square array fiber structures, at different porosities. This numerical data was then used to derive the moments $\langle\delta\rangle$ and $\left\langle\delta^{2}\right\rangle$, mean survival time, $\tau$, and principal relaxation time, $T_{1}$, and test the accuracy of variational bounds and a universal scaling expression.

The dimensionless mean survival time and principal relaxation time were found to increase with porosity, remain practically independent of the directionality of random fiber beds, and attain lower values for ordered arrays. The dimensionless mean pore size, $\langle\delta\rangle / r$, and mean square pore size, $\left\langle\delta^{2}\right\rangle / r^{2}$, were found to follow the same general trends, too, with regards to the porosity, directionality, and order effects. The corresponding pore size probability distributions were found typically wide for random fiber structures, but narrow for square arrays, and in very good agreement with theoretically predicted limiting values at $\delta=0$, for all types of structures. For the square array of fibers, we derived analytical results for the pore size probability and its moments, which were found to practically coincide with the corresponding numerical results in all cases.

The variational lower bounds $\tau \geqslant\langle\delta\rangle^{2} / D$ and $T_{1}$ $\geqslant\left\langle\delta^{2}\right\rangle / D$ are obeyed by our simulation results in all cases, and are quite sharp up to very high porosities, in agreement with earlier predictions. The bound $\tau \leqslant T_{1}$ is also obeyed by our numerical results for all random and ordered arrays of fibers, becoming an equality at the dilute limit. For all random and ordered fiber structures, the values of the ratios $\left\langle\delta^{2}\right\rangle /\langle\delta\rangle^{2}, \tau D /\langle\delta\rangle^{2}, T_{1} D /\left\langle\delta^{2}\right\rangle$, and $T_{1} / \tau$ were found to vary in practically the same range as the corresponding ratios reported earlier for beds of spherical particles, revealing some fundamental similarity in the transport and structural properties of these otherwise very different families of porous media.

A universal curve relating the mean survival time to the mean pore size through properly defined length and time scales, derived earlier for various types of sphere assem- blages, was found to agree throughout the porosity range with our numerical data for random fiber structures, and up to a very high porosity $(\varepsilon \approx 0.9)$ for the square array. This is the first validation of this scaling expression for anisotropic porous media, lending support to the claim that it describes a broad variety of porous structures.

\section{ACKNOWLEDGMENT}

This work was supported in part by a grant from the Florida Solar Energy Center.

${ }^{1}$ M. M. Tomadakis and S. V. Sotirchos, J. Chem. Phys. 98, 616 (1993).

${ }^{2}$ M. M. Tomadakis and S. V. Sotirchos, J. Chem. Phys. 99, 9820 (1993).

${ }^{3}$ M. M. Tomadakis and S. V. Sotirchos, J. Chem. Phys. 104, 6893 (1996).

${ }^{4}$ M. M. Tomadakis and S. V. Sotirchos, J. Chem. Phys. 109, 4508 (1998); AIChE J. 39, 397 (1993); 37, 74 (1991); 37, 1175 (1991); Radiat. Res. 135, 302 (1993).

${ }^{5}$ P. R. Johnston, Filtr. Sep. 35, 287 (1998); H. W. Piekaar and L. A. Clarenburg, Chem. Eng. Sci. 22, 1399 (1967); H. K. Corte and E. H. Lloyd, in Consolidation of the Paper Web (British Paper and Board Makers' Association, London, 1966), pp. 981-1009.

${ }^{6}$ E. A. Efthimiadis and S. V. Sotirchos, Chem. Eng. Sci. 48, 1971 (1993); S. Zarkanitis and S. V. Sotirchos, AIChE J. 35, 821 (1989); S. V. Sotirchos and H. C. Yu, Chem. Eng. Sci. 40, 2039 (1985).

${ }^{7}$ S. Prager, Chem. Eng. Sci. 18, 227 (1963).

${ }^{8}$ D. A. Coker and S. Torquato, J. Appl. Phys. 77, 6087 (1995); C. L. Y. Yeong and S. Torquato, Phys. Rev. E 58, 224 (1998); M. D. Rintoul and S. Torquato, J. Colloid Interface Sci. 186, 467 (1997).

${ }^{9}$ G. A. Coker, S. Torquato, and J. H. Dunsmuir, J. Geophys. Res. 101, 17497 (1996); M. D. Rintoul, S. Torquato, C. Yeong, D. T. Keane, S. Erramilli, Y. N. Jun, D. M. Dabbs, and I. A. Aksay, Phys. Rev. E 54, 2663 (1996); C. Manwart, S. Torquato, and R. Hilfer, ibid. 62, 893 (2000).

${ }^{10}$ S. Torquato and M. Avellaneda, J. Chem. Phys. 95, 6477 (1991).

${ }^{11}$ D. A. Coker and S. Torquato, J. Appl. Phys. 77, 955 (1995).

${ }^{12}$ H. P. G. Drewry and N. A. Seaton, AIChE J. 41, 880 (1995).

${ }^{13}$ S. Torquato and I. C. Kim, J. Appl. Phys. 72, 2612 (1992).

${ }^{14}$ S. Torquato and C. L. Y. Yeong, J. Chem. Phys. 106, 8814 (1997).

${ }^{15}$ G. Srinivas, K. L. Sebastian, and B. Bagchi, J. Chem. Phys. 116, 7276 (2002); J. Sung, E. Barkai, R. J. Silbey, and S. Lee, ibid. 116, 2338 (2002); H.-X. Zhou, ibid. 108, 8139 (1998).

${ }^{16}$ N. Agmon, J. Chem. Phys. 81, 3644 (1984).

${ }^{17}$ J. R. Banavar and L. M. Schwartz, Phys. Rev. Lett. 58, 1411 (1987).

${ }^{18}$ K. J. Dunn, G. A. LaTorraca, and D. J. Bergman, Geophysics 64, 470 (1999); A. Viallat and M. M. Margulies, Eur. Phys. J. E 2, 117 (2000); W. E. Kenyon, P. I. Day, C. Straley, and J. F. Willemsen, SPE Formation Evaluation, September, 1988, p. 622.

${ }^{19}$ G. C. Borgia, V. Bortolotti, A. Brancolini, R. J. S. Brown, and P. Fantazzini, Magn. Reson. Imaging 14, 751 (1996).

${ }^{20}$ S. Torquato, Phys. Rev. Lett. 64, 2644 (1990); M. Avellaneda and S. Torquato, Phys. Fluids A 3, 2529 (1991); L. M. Schwartz, N. Martys, D. P. Bentz, E. J. Garboczi, and S. Torquato, Phys. Rev. E 48, 4584 (1993).

${ }^{21}$ A. E. Scheidegger, The Physics of Flow Through Porous Media (University of Toronto Press, Toronto, 1957), p. 7.

${ }^{22}$ R. Coleman, J. Appl. Probab. 6, 430 (1969).

${ }^{23}$ M. M. Tomadakis, Ph.D. thesis, University of Rochester, Rochester, 1993.

${ }^{24}$ C. David, J. Geophys. Res., [Solid Earth] 98, 12267 (1993).

${ }^{25}$ M. M. Tomadakis and T. J. Robertson, Phys. Rev. B (to be published). 